\title{
Air Pollution Reduction Based on Intelligent Nonlinear Control Methodology
}

\author{
Yaser Barmayeh, Mehran Afshar, Mohammad Sadegh Dahideh, Mohammad \\ Najafi, and Ali Reza Zarei \\ Department of Electrical Engineering, Delvar Branch, Islamic Azad University, \\ Delvar, Iran \\ Yaser.barmayeh2014@gmail.com
}

\begin{abstract}
This paper expands a Multi Input Multi Output (MIMO) fuzzy baseline control (FBC) which controller coefficient is off-line tuned by gradient descent algorithm. The main goal is to adjust the optimal value for fuel ratio $(F R)$ in motor engine. The fuzzy inference system in proposed methodology is works based on Mamdani-Lyapunov fuzzy inference system (FIS). To reduce dependence on the gain updating factor coefficients of the fuzzy methodology, PID baseline method is introduced. This new method provides an optimal setting for other factors which created by PID baseline method. The gradient descent methodology is off-line tune all coefficients of baseline fuzzy based on mathematical optimization methodology. Simulation results signify good performance of fuel ratio in presence of different torque load and external disturbance.
\end{abstract}

Keywords: Baseline method, Gradient descent optimal algorithm, fuzzy inference system, Lyapunov based stability

\section{Introduction}

The internal combustion (IC) engine is designed to produce power from the energy that is contained in its fuel. More specifically, its fuel contains chemical energy and together with air, this mixture is burned to output mechanical power [1-3]. In an internal combustion engine, a piston moves up and down in a cylinder and power is transferred through a connecting rod to a crank shaft. The continual motion of the piston and rotation of the crank shaft as air and fuel enter and exit the cylinder through the intake and exhaust valves is known as an engine cycle. The fuel ratio can be used to determine which fuel system should have a larger impact on how much fuel is injected into the cylinder [4-9]. Since a direct fuel injector (DI) has immediate injection of its fuel with significant charge cooling effect, it can have a quicker response to the desired amount of fuel that is needed by an engine. Although a port fuel injector (PFI) may have a slower response due to its wall-wetting dynamics, the fuel ratio will impact the combustion characteristics of an engine. Fuel ratio also can be used to regulate or control two fuel types. For example, an engine may have the ability to run on gasoline and ethanol. The gasoline could be injected by a PFI, while the ethanol could be injected by a DI [1-9].

The controller is a device which can sense information from linear or nonlinear system (e.g., IC engine) to improve the systems performance [3]. The main targets to design a control systems are stability, good disturbance rejection, and small tracking error [5]. In automotive industries several IC engines are controlled by linear methodologies (e.g., Proportional-Derivative (PD) methodology, Proportional- Integral (PI) method or Proportional- Integral-Derivative (PID) method), but it has many limitation to have a good result. In some applications IC engines are used in an unknown and unstructured environment, therefore strong mathematical tools used in new control methodologies to design fuzzy plus PD controller based on computed fuel ratio compensation [4-5]. 
Fuzzy-logic aims to provide an approximate but effective means of describing the behavior of systems that are not easy to describe precisely, and which are complex or illdefined [5-17]. It is based on the assumption that, in contrast to Boolean logic, a statement can be partially true (or false) [6-7]. For example, the expression (I live near Boushehr Islamic Azad University) where the fuzzy value (near) applied to the fuzzy variable (distance), in addition to being imprecise, is subject to interpretation. The essence of fuzzy control is to build a model of human expert who is capable of controlling the plant without thinking in terms of its mathematical model. As opposed to conventional control approaches where the focus is on constructing a controller described by differential equations, in fuzzy control the focus is on gaining an intuitive understanding (heuristic data) of how to best control the process [13], and then load this data into the control system [10-11].

Normal combinations of PD control with fuzzy logic (PD+FL) is design to automotive engine [12], while FLC compensates the control error, baseline methodology reduces remain error of fuzzy PD to have robust and stable result [17-19]. The error is reducing, because baseline methodology and fuzzy logic controller work parallel.

This paper is organized as follows; second part focuses on the modeling dynamic formulation based on Lagrange methodology, fuzzy logic methodology and linear controller are discussion. Third part is focused on the methodology which can be used to reduce the error, increase the performance quality and increase the robustness and stability. Simulation result and discussion is illustrated in forth part which based on trajectory following and disturbance rejection. The last part focuses on the conclusion and compare between this method and the other ones.

\section{Theory}

\section{A. Dynamic Formulation of IC Engine}

Dynamic modeling of IC engine is used to describe the nonlinear behavior of IC engine, design of model based controller such as pure variable structure controller based on nonlinear dynamic equations, and for simulation. The dynamic modeling describes the relationship between fuel to air ratio to PFI and DI and also it can be used to describe the particular dynamic effects (e.g., motor pressure, angular speed, mass of air in cylinder, and the other parameters) to behavior of system [1].

The equation of an IC engine governed by the following equation [1]:

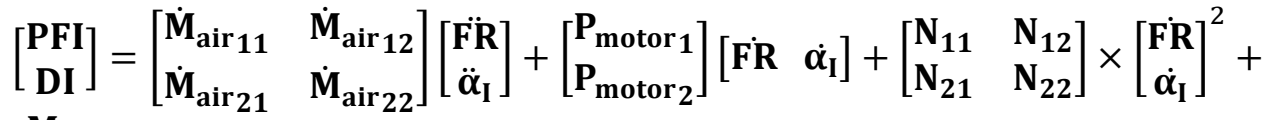

$$
\begin{aligned}
& {\left[\begin{array}{l}
\mathbf{M}_{\mathrm{a}_{1}} \\
\mathbf{M}_{\mathrm{a}_{2}}
\end{array}\right]}
\end{aligned}
$$

Where $P F I$ is port fuel injector, $D I$ is direct injector, $\dot{\mathbf{M}}_{\mathrm{air}}$ is a symmetric and positive define mass of air matrix, $\mathbf{P}_{\text {motor }}$ is the pressure of motor, $N$ is engine angular speed and $\mathbf{M}_{\mathbf{a}}$ is matrix mass of air in cylinder. Fuel ratio and exhaust angle are calculated by:

$$
\begin{aligned}
& {\left[\begin{array}{c}
F \ddot{R}_{a} \\
\ddot{\alpha}_{I_{a}}
\end{array}\right]=\left[\begin{array}{ll}
\dot{M}_{\text {air } 11} & \dot{M}_{\text {air } 12} \\
\dot{M}_{\text {air } 21} & \dot{M}_{\text {air } 22}
\end{array}\right]^{-1}\left\{\left[\begin{array}{c}
P F I \\
D I
\end{array}\right]-\left\{\left[\begin{array}{l}
P_{\text {motor }_{1}} \\
P_{\text {motor }_{2}}
\end{array}\right]\left[\begin{array}{ll}
F R & \dot{\alpha}_{I a}
\end{array}\right]+\left[\begin{array}{ll}
N_{11} & N_{12} \\
N_{21} & N_{22}
\end{array}\right] \times\right.\right.} \\
& \left.\left.\left[\begin{array}{c}
F \dot{R}_{a} \\
\dot{\alpha}_{I_{a}}
\end{array}\right]^{2}+\left[\begin{array}{c}
M_{a_{1}} \\
M_{a_{2}}
\end{array}\right]\right\}\right\}
\end{aligned}
$$


The above target equivalence ratio calculation will be combined with fuel ratio calculation that will be used for controller design purpose.

\section{B. Control Theory}

The design of a baseline methodology to control the fuel ratio was very straight forward. Since there was an output from the fuel ratio model, this means that there would be two inputs into the baseline controller. Similarly, the output of the controller result from the two control inputs of the port fuel injector signal and direct injector signal. In a typical PID method, the controller corrects the error between the desired input value and the measured value. Since the equivalence ratio and fuel ratio are the two measured signals, two controllers were cascaded together to control the PFI and DI inputs. The first was a PID controller that corrected the error between the desired equivalence ratio and the measured equivalence ratio; while the second was only a proportional integral (PI) controller that corrected the fuel ratio error.

$$
\begin{aligned}
& e_{1}(t)=\alpha_{\text {target }}(t)-\alpha_{d}(t) \\
& e_{2}(t)=\text { Fuel ratio } a(t)-\text { Fuel Ratio }{ }_{d}(t) \\
& P F I_{\alpha}=K_{p_{a}} e_{1}+K_{V_{a}} \dot{e_{1}}+K_{I_{a}} \sum e_{1} \\
& D I_{\alpha}=K_{p_{b}} e_{1}+K_{V_{b}} e_{1}+K_{I_{b}} \sum e_{1} \\
& P F I_{F}=\left(K_{p_{c}} e_{2}+K_{I_{c}} \sum e_{2}\right) \times P F I_{\alpha} \\
& D I_{F}=D I_{\alpha}
\end{aligned}
$$

Based on foundation of fuzzy logic methodology; fuzzy logic controller has played important rule to design nonlinear controller for nonlinear and uncertain systems [15-17]. However the application area for fuzzy control is really wide, the basic form for all command types of controllers consists of;

Input fuzzification (binary-to-fuzzy $[\mathrm{B} / \mathrm{F}]$ conversion) Fuzzy rule base (knowledge base), Inference engine and Output defuzzification (fuzzy-to-binary [F/B] conversion). The fuzzy inference engine offers a mechanism for transferring the rule base in fuzzy set which it is divided into two most important methods, namely, Mamdani method and Sugeno method. Mamdani method is one of the common fuzzy inference systems and he designed one of the first fuzzy controllers to control of system engine. Mamdani's fuzzy inference system is divided into four major steps: fuzzification, rule evaluation, aggregation of the rule outputs and defuzzification. Michio Sugeno use a singleton as a membership function of the rule consequent part. The following definition shows the Mamdani and Sugeno fuzzy rule base

\section{if $x$ is $A$ and $y$ is $B$ then $z$ is $C$ 'mamdani' if $x$ is $A$ and $y$ is $B$ then $z$ is $f(x, y)$ 'sugeno'}

When $x$ and $y$ have crisp values fuzzification calculates the membership degrees for antecedent part. Rule evaluation focuses on fuzzy operation $(A N D / O R)$ in the antecedent of the fuzzy rules. The aggregation is used to calculate the output fuzzy set and several methodologies can be used in fuzzy logic controller aggregation, namely, Max-Min aggregation, Sum-Min aggregation, Max-bounded product, Max-drastic product, Max- 
bounded sum, Max-algebraic sum and Min-max. Two most common methods that used in fuzzy logic controllers are Max-min aggregation and Sum-min aggregation.

Defuzzification is the last step in the fuzzy inference system which it is used to transform fuzzy set to crisp set. Consequently defuzzification's input is the aggregate output and the defuzzification's output is a crisp number. Centre of gravity method $(C O G)$ and Centre of area method $(C O A)$ are two most common defuzzification methods.

\section{Methodology}

In this method fuzzy logic method is used to estimate some unknown dynamic formulation. Above methods have acceptable performance based on dynamic modeling of IC engines but this research is focused on eliminate the nonlinear IC engine's dynamic formulation. In this method; nonlinear dynamic model based part is replaced by performance/error-based Mamdani's fuzzy inference system. It has considered one input; variable structure surface $(S)$, one output; $P F I / D I$ and totally 7 rules instead of the nonlinear dynamic model part. This method has an important challenge to adjust the variable structure slope $(\lambda)$. To solve above challenge new based-line method is applied to fuzzy methodology to reduce the role of initial value of $\lambda$ in fuzzy methodology.

Based on fuzzy logic methodology

$f(x)=U_{f u z z y}=\sum_{l=1}^{M} \theta^{T} \zeta(x)$

where $\boldsymbol{\theta}^{\boldsymbol{T}}$ is adjustable parameter (gain updating factor) and $\boldsymbol{\zeta}(\boldsymbol{x})$ is defined by

$\zeta(x)=\frac{\sum_{i} \mu\left(x_{i}\right) x_{i}}{\sum_{i} \mu\left(x_{i}\right)}$

Where $\boldsymbol{\mu}\left(\boldsymbol{x}_{\boldsymbol{i}}\right)$ is membership function. $\boldsymbol{P F} \boldsymbol{I}_{\boldsymbol{f u z z} \boldsymbol{y}}$ is defined as follows;

$P F I_{\text {fuzzy }}=\sum_{l=1}^{M} \theta^{T} \zeta(x)=\left[\dot{M}^{-1}\right.$ air $\left(\left[\begin{array}{l}P_{\text {motor }_{1}} \\ P_{\text {motor }_{2}}\end{array}\right]\left[\begin{array}{ll}F R & \dot{\alpha}_{I}\end{array}\right]+\left[\begin{array}{ll}N_{11} & N_{12} \\ N_{21} & N_{22}\end{array}\right] \times\right.$

$\left.\left.\left[\begin{array}{c}\dot{F R} \\ \dot{\alpha_{I}}\end{array}\right]^{2}+\left[\begin{array}{c}M_{a_{1}} \\ M_{a_{2}}\end{array}\right]\right)+\dot{S}\right] \dot{M}_{a i r}$

Design fuzzy like nonlinear part has the following steps;

Fuzzification: in this step the researcher must to defined the linguistic variables for input, $(S)$, and outputs, (PFI and DI). This research defined 7 linguistic variables for input and output which names; Negative Big (NB), Negative Medium (NM), Negative Small (NS), Zero (ZE), Positive Small (PS), Positive Medium (PM) and Positive Big (PB). Triangular membership function is used to design this controller. Based on this membership function it can guarantee the output performance. Input and output are quantized into thirteen levels represented by: $-6,-5,-0.4,-3,-2,-1,0,1,2,3,4,5,6$.

Fuzzy rule base and rule evaluation: this design has 7 rules which design by researcher's experience knowledge. Design the rule base of fuzzy inference system can play important role to design the best performance of fuzzy VSC, that to calculate the fuzzy rule base the researcher is used to heuristic method which, it is based on the behavior of the control of IC engine.

Rule evaluation focuses on operation in the antecedent of the fuzzy rules in fuzzy controller. In this research, researcher is used $A N D$ fuzzy operation in antecedent part. After calculate rule evaluation, activation degree applied to antecedent part and connect it 
to consequent part based on $A N D$ operation in antecedent part. Finally rule aggregation is introduced which, Max-Min aggregation is used in this paper.

Inference Mechanism: in this paper Mamdani's fuzzy inference mechanism is used based on eliminate the IC engine's dynamic formulation.

Defuzzification: the final steps to design fuzzy controller is select the defuzzification methodology. In this paper Center of Gravity method $(C O G)$ is used to transform fuzzy to crisp.

The most important coefficient in proposed fuzzy methodology is, variable surface slope. Predefined this coefficient is complicated; therefore this research is used PID baseline method and applied to fuzzy logic methodology. PID baseline methodology is tune based on three coefficient; namely $\boldsymbol{K}_{\boldsymbol{p}}, \boldsymbol{K}_{\boldsymbol{v}}, \boldsymbol{K}_{\boldsymbol{i}}$. One of the positive effects of these three coefficients is tune the PFI easily. Proposed fuzzy controller are tuned based on variable structure surface slope $(\boldsymbol{\lambda})$ that tune it to have best performance is very complicated. For instance, if large value of $\lambda$ is chosen the response is very fast the system is unstable and conversely, if small value of $\lambda$ is considered the response of system is very slow but system is stable. Therefore to have a good response, compute the best value variable structure slope coefficient is very important. To adjust this coefficient Gradient Descent optimization is introduced which this method is worked based on the observation methodology. To easy tuning PID baseline methodology is used. When this method is applied to fuzzy methodology the number of coefficient are increases but tune them are better than previous.

\section{Results and Discussion}

This simulation begins using a uniform random number for the air input and adds a step input of 0.15 at 6 seconds. The random number had a minimum value of -0.15 , a maximum value of 0.15 , and the value changed to a number between these two extremes every time it was sampled.

The closed loop response of the system in this research although there was fluctuating random air flow disturbance throughout the entire response. The controller achieved and maintained the desired ratios but due to the random input disturbances could not maintain a smooth response. Figure 1 shows the close loop response of equivalence ratio and fuel ratio.

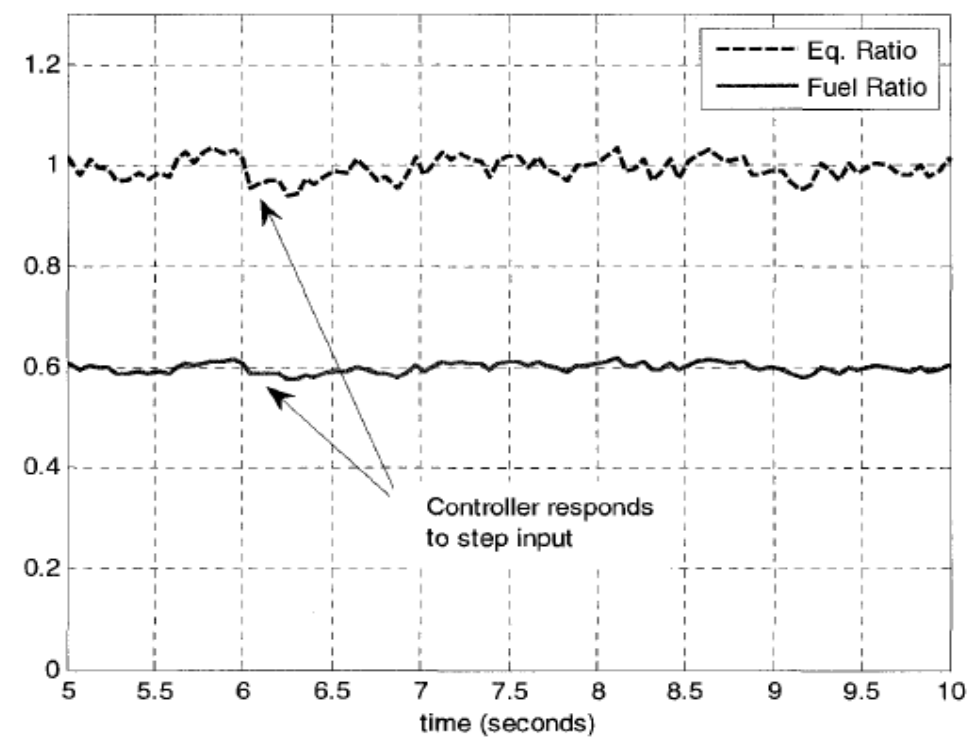

Figure 1. Equivalence Ratio and Fuel Ratio 
The control inputs in this part were under variations in its signal. The control efforts of PFI and DI achieved the desired ratio but its variations caused fluctuations in the desired $\mathrm{A} / \mathrm{F}$ and fuel ratios. These fluctuations corresponded to the changing random number that was modeled as the air input.

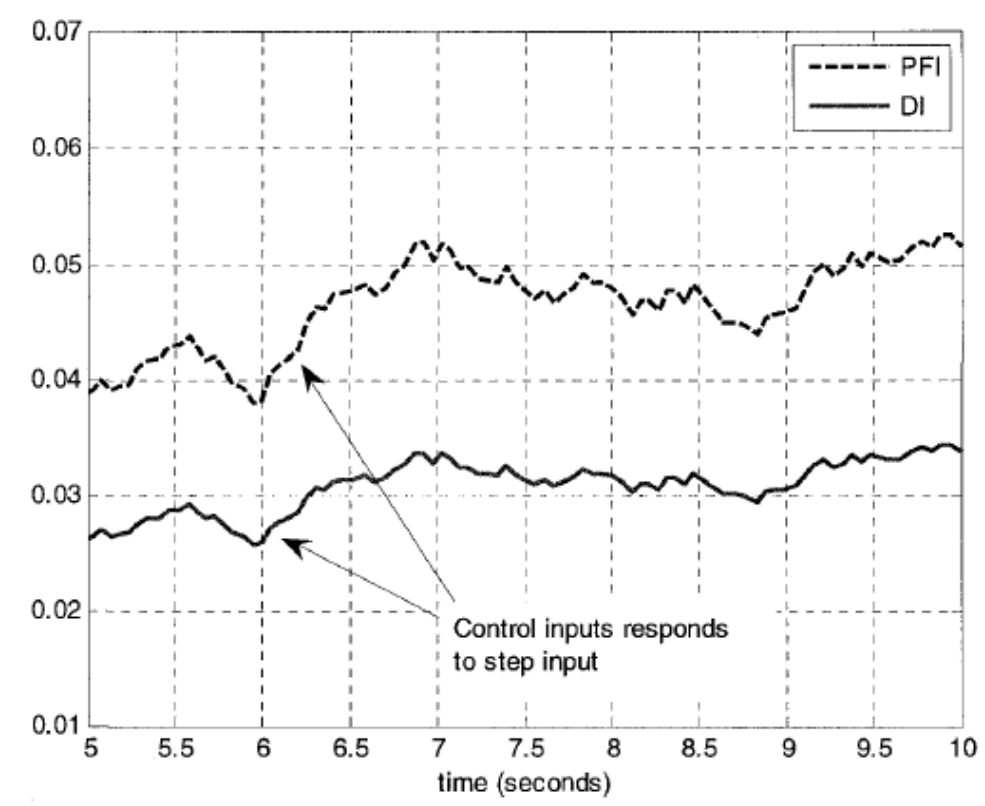

Figure 2. PFI and DI Control Inputs

\section{Conclusion}

Refer to this research, design an artificial intelligence based nonlinear methodology is proposed to tune the fuel ratio in IC engine. The mean-value engine model can be used to validate control strategies for different types of controllers that are model-based. The equations that are used to calculate the outputs of the model are approximated over an engine cycle. A significant advantage of the mean-value engine model is its low computational throughput which makes it possible for real-time simulation. In order to reduce engine emissions and improving engine fuel economy closed loop combustion control, which requires cycleto-cycle combustion measurement such as cylinder pressure, is a necessity.

The nonlinear controller is a multi-input and multi-output (MIMO). Proposed controller, which had two inputs, equivalence ratio and fuel ratio, and two outputs, PFI and DI fueling, is useful for $\mathrm{A} / \mathrm{F}$ ratio and fuel ratio control. This is primarily due to the fact that PID controllers are widely used in automotive applications and can be easily implemented. This controller is depending on variable structure slope that to solve this challenge baseline methodology is applied to fuzzy variable structure method. Eventually gradient descent optimization is applied to fuzzy baseline methodology to tune all four parameters. The fuel ratio results demonstrate that the gradient descent fuzzy baseline methodology is a model-free methods which works well in certain and partly uncertain system.

\section{References}

[1] J. Heywood, "Internal Combustion Engine Fundamentals", McGraw-Hill, New York, (1988).

[2] J. G. Rivard, "Closed-loop Electronic Fuel Injection Control of the IC Engine," in Society of Automotive Engineers, (1973).

[3] J. F. Cassidy, "On the Design of Electronic Automotive Engine Controls using linear Quadratic Control Theory," IEEE Trans on Control Systems, vol. AC-25, (1980) October. 
[4] W. E. Powers, "Applications of Optimal Control and Kalman Filtering to Automotive Systems," International Journal of Vehicle Design, vol. Applications of Control Theory in the Automotive Industry, (1983).

[5] N. F. Benninger, "Requirements and Perfomance of Engine Management Systems under Transient Conditions," in Society of Automotive Engineers, (1991).

[6] N. F. Benninger, "Requirements and Perfomance of Engine Management Systems under Transient Conditions," in Society of Automotive Engineers, (1991).

[7] C. H. Onder, "Model-Based Multivariable Speed and Air-to-Fuel Ratio Control of an SI Engine," in Society of Automotive Engineers, (1993).

[8] S. B. Cho, "An Observer-based Controller Design Method for Automotive Fuel-Injection Systems," in American Controls Conference, (1993), pp. 2567-2571.

[9] T. Kume, "Combustion Technologies for Direct Injection SI Engine," in Society of Automotive Engineers, (1996).

[10] V. Utkin, "Variable structure systems with sliding modes," Automatic Control, IEEE Transactions on, vol. 22, no. 2, (2002), pp. 212-222.

[11] R. A. DeCarlo, S. H. Zak and G. P. Matthews, "Variable structure control of nonlinear multivariable systems: a tutorial," Proceedings of the IEEE, vol. 76, no. 3, (2002), pp. 212-232.

[12] K. D. Young, V. Utkin and U. Ozguner, "A control engineer's guide to sliding mode control," IEEE conference proceeding, (2002), pp. 1-14.

[13] O. Kaynak, "Guest editorial special section on computationally intelligent methodologies and slidingmode control," IEEE Transactions on Industrial Electronics, vol. 48, no. 1, (2001), pp. 2-3.

[14] F. Piltan, M. Akbari, M. Piran and M. Bazregar, "Design Model Free Switching Gain Scheduling Baseline Controller with Application to Automotive Engine", International Journal of Information Technology and Computer Science, vol. 5, no. 1, (2013), pp. 65-73.DOI: 10.5815/ijitcs.2013.01.07. (DOAJ, DOI: 10.5815)

[15] P. Kachroo and M. Tomizuka, "Chattering reduction and error convergence in the sliding-mode control of a class of nonlinear systems," Automatic Control, IEEE Transactions on, vol. 41, no. 7, (2002), pp. 1063-1068.

[16] J. Moura and N. Olgac, "A comparative study on simulations vs. experiments of SMCPE," IEEE conference proceeding, (2002), pp. 996-1000.

[17] F. Piltan, N. Sulaiman, P. Ferdosali and I. A. Talooki, "Design Model Free Fuzzy Sliding Mode Control: Applied to Internal Combustion Engine", International Journal of Engineering, vol. 5, no. 4, (2011), pp. 302-312.

[18] F. Piltan, N. Sulaiman, I. A. Talooki and P. Ferdosali, "Control of IC Engine: Design a Novel MIMO Fuzzy Backstepping Adaptive Based Fuzzy Estimator Variable Structure Control ," International Journal of Robotics and Automation, vol. 2, no. 5, (2011), pp. 360-380.

[19] F. Piltan, M. Piran, M. Bazregar and M. Akbari, "Design High Impact Fuzzy Baseline Variable Structure Methodology to Artificial Adjust Fuel Ratio", International Journal of Intelligent Systems and Applications, vol. 5, no. 2, (2013), pp. 59-70. DOI: 10.5815/ijisa.2013.02.0. (DOAJ, DOI: 10.5815) 
International Journal of Hybrid Information Technology Vol.8, No.1 (2015) 